\title{
A comparison between Pap and HPV screening tests and screening methods
}

\author{
EMMA ALTOBELLI ${ }^{1,2}$, GIORGIO SCARSELLI ${ }^{2}$, AMEDEO LATTANZI ${ }^{2}$, \\ CARMINE FORTUNATO $^{3}$ and VALERIO F. PROFETA ${ }^{4}$ \\ ${ }^{1}$ Department of Life, Health and Environmental Sciences, University of L'Aquila, I-67100 L'Aquila; \\ ${ }^{2}$ Epidemiology and Statistics Unit, Departments of ${ }^{3}$ Pathological Anatomy \\ and ${ }^{4}$ Community Health, AUSL 4, I-67100 Teramo, Italy
}

Received May 11, 2015; Accepted March 3, 2016

DOI: $10.3892 / \mathrm{mco} .2016 .909$

\begin{abstract}
The present study assesses the results of cervical cancer (CC) screening over two 3-year periods (2008-2010 and 2011-2013) by comparing two screening tests [Papanicolaou (Pap) and human papillomavirus (HPV) tests] and two screening methods (organized and spontaneous). The study population includes women aged 25-64 years who underwent CC screening between 2008 and 2010 and/or 2011 and 2013, divided into those who responded to an invitation letter (organized screening) and those who spontaneously underwent testing at a public or private facility (non-programmed screening). Between 2008 and 2010, the response rates increased from $27.7 \%$ in 2008 to $44.5 \%$ in 2009 and $67.6 \%$ in $2010(\mathrm{P}<0.001)$. Women aged $25-34$ years had the lowest response rate, whereas respondents were more frequent among women aged 35-44 and 45-54 years. Significant differences $(\mathrm{P}<0.001)$ were identified between organized and spontaneous screening test results with regard to diagnostic categories high-grade squamous intraepithelial lesion (50.5 vs. $49.5 \%$ ), low-grade squamous intraepithelial lesion (42.8 vs. $57.2 \%$ ) and undetermined lesion atypical glandular cells (AGC; 57.5 vs. $42.5 \%$ ) or atypical squamous cells of undetermined significance (ASC-US; 54.2 vs. 45.8\%). Compared with spontaneous screening, the organized programme resulted in a larger number of women screened for $\mathrm{CC}$; it reduced the frequency of undetermined diagnoses (AGC, ASC-US), and identified a larger number of high-grade lesions.
\end{abstract}

\section{Introduction}

Cervical cancer (CC) is the second most frequent cancer and the third cause of fatality for women worldwide. However, $~ 85 \%$ of

Correspondence to: Professor Emma Altobelli, Department of Life, Health and Environmental Sciences, University of L'Aquila, Piazzale Salvatore Tommasi, 1 Coppito, I-67100 L'Aquila, Italy E-mail: emma.altobelli@cc.univaq.it

Key words: cancer, cervical cancer, screening, Papanicolaou test, human papillomavirus test all CC cases are diagnosed in less-developed countries (1). It has been estimated that in 2012 there were 33,000 new CC cases and 13,000 mortalities across the European Union (2). According to incidence estimates from the Italian Cancer Registry Association, 2,000 new cases arose in Italy in 2013 (3). While 351 CC-related fatalities were reported to the Italian National Institute of Statistics in 2006, this figure is possibly underestimated by as much as $60 \%$ (4). Furthermore, these figures indicate a marked geographic trend, with a 10 and $30 \%$ higher mortality in central and southern Italy, respectively, compared with the north of the country (5). In Italy, the Papanicolaou (Pap) test for spontaneous screening has occurred since the 1960s and, in particular, the adoption of organized screening programmes over the past two decades has been the main factor in the reduction in CC incidence and mortality. According to 2012 data, region-based screening programmes currently cover $84 \%$ of the female population aged 25-64 years (6), with $77 \%$ of women receiving an invitation letter; the response rate is $\sim 43 \%$ of the female population of Italy (7). The recent Health Technology Assessment and the recommendations by the Italian National Screening Observatory's guidance document (6) have led to the replacement of the Pap test with the human papillomavirus (HPV) test as the primary screening test in several Italian programmes, including that of Abruzzo, a region in central Italy. Italy and The Netherlands were the first EU member States to adopt this radical change (8). In Abruzzo, CC screening with the HPV test commenced in January 2011, and the first screening round with this method was completed in December 2013. Over the same period, new provisions regulating access to the free organized programme have been introduced to reduce spontaneous screening, as only women who have been invited by the Programme can undergo a free screening test. In the present study, the results obtained over two screening periods (2008-2010 and 2011-2013) were assessed by comparing two CC screening tests (Pap and HPV test) and two screening methods (organized and spontaneous) in the population covered by the Local Health Care Unit (AUSL) of Teramo, Abruzzo.

\section{Patients and methods}

Population. The study population consisted of women aged 25-64 years residing in Teramo province who underwent CC 
screening between 2008 and 2010 and/or 2011 and 2013, and whose test was examined at the pathology service of Atri or Teramo hospitals in line with the local screening protocol, as indicated below. The population was further divided into women who responded to an invitation letter (programmed screening) and those who underwent spontaneous testing at a public or private laboratory (spontaneous screening). The target population consisted of 85,289 women between 2008 and 2010 and 85,764 between 2011 and 2013 (mean resident population) (9).

Screening programme exclusion criteria. Women aged $<25$ and $>64$ years, those who had previously undergone a hysterectomy and those suffering from cancer involving the cervix were not invited to screen. Women who had been previously treated for a cervical tumour or for histological preneoplastic (CIN) lesions, with partial sparing of the cervix were followed up at 6-or 12-month intervals, according to lesion severity.

Spontaneous screening. A Pap test can be obtained at a public facility or a private gynaecological practice. Until January 2011, any woman could present spontaneously at a public facility for a free Pap test at any time; women in the screening roll call could also go during the 3 -year interval between rounds. When samples were examined by one of the two aforementioned pathology services they were archived in the AUSL database. Analysis of these data together with those from organized screening provides a more accurate depiction of the coverage of the target population for CC prevention.

Screening protocol. In 2011, two notable changes were added to the Teramo AUSL protocol: The Pap test was replaced with the HPV test, which is free only for women who respond to an invitation letter from the Programme in order to reduce spontaneous participation. In the screening protocol an invitation to screen stating an appointment date and time is sent to all eligible women who have not undergone CC screening in the previous 3 years. Women who do not present for the test are sent a reminder within 6 months. Until 2010 the protocol envisaged a Pap smear as the first-level screening test for organized as well as spontaneous testing. In 2011, the regional government of Abruzzo started a project based on DNA testing for high-risk oncogenic HPV types by the Hybrid Capture 2 assay as the primary screening test. Women with negative results are referred to the subsequent screening round, whereas positive samples are sent for cytology. The liquid-phase assay enables HPV testing and cytology triage (10). To ensure consistently high-quality analysis, all test samples are examined at a single, accredited reference centre in the province, where test reading and reporting are performed by automated procedures. Positive samples undergo cytology triage with the Pap test. When the Pap test is negative, the HPV and Pap test reports are sent to the woman, who is informed that a recall will occur at a 1-year interval for a new test. When the Pap test is positive or equivocal, the woman is contacted by phone for second-level colposcopy. For the patients involved in the present study, high-quality examination was ensured by accreditation of a single gynaecology department in the province to perform all screening colposcopies and biopsy examinations (where required). Spontaneous testing at a private facility involves only a conventional Pap test.

Diagnostic categories. Cytological diagnosis of the Pap test as a screening or triage test uses the 2001 Bethesda classification system (11) and the following diagnostic categories: Negative for intraepithelial lesion or malignancy (negative); atypical squamous cells of undetermined significance (ASC-US); atypical squamous cells of undetermined significance, which cannot exclude high-grade squamous intraepithelial lesion (ASC-H); atypical glandular cells (AGC); low-grade squamous intraepithelial lesion (LSIL); high-grade squamous intraepithelial lesion (HSIL); and squamous cell carcinoma. The histological diagnoses are listed as 'non-dysplastic (negative) lesions', 'cervical intraepithelial neoplasia low (CIN 1), moderate (CIN 2), severe (CIN 3)', carcinoma and adenocarcinoma.

Data sources. Case ascertainment was based on the regional electronic information system (SIW) and the AUSL pathology information system (SIA). The former system manages target population demographics, the invitations of eligible women, the link between samples and the invitation letter, and HPV and Pap test reports. The SIA manages the results of second-level tests (colposcopies and biopsy examinations), of spontaneous tests analysed by the two accredited laboratories, and of the tests undergone by women treated for an earlier $\mathrm{CC}$, who are examined at short intervals.

Statistical analysis. Continuous variables are presented as mean \pm standard deviation. The Pearson $\chi^{2}$ test was used to compare the distribution of categorical variables. $\mathrm{P}<0.05$ was considered to indicate a statistically significant difference. All analyses were carried out using Stata 11 SE statistical software (Stata Corp LP, College Station, TX, USA).

\section{Results}

Screening participation. Over the 6 years examined, organized screening accounted for 38,348 tests and spontaneous screening for 21,140. The organized screening data, with the number of invitations and response rates, are reported in Table I.

Between 2008 and 2010, a total of 36,581 women (40.5\% of the target population) were sent an invitation to be screened and 14,142 responded; their mean age was $44.5 \pm 10.5$ years. Between 2011 and 2013, a total of 60,118 women (70.1\% of the target population) were invited and 24,206 responded; their mean age was $44.5 \pm 10.6$ years. Data analysis highlighted that over the first 3-year period, the response rate increased significantly from $27.7 \%$ in 2008 to $44.6 \%$ in 2009 and $51.4 \%$ in $2010(\mathrm{P}<0.001)$. In the second period, the response rate increased only in 2012 , to $43.8 \%$, and subsequently declined to $42.3 \%$ in 2013, despite more invitations being sent to patients $(\mathrm{P}<0.001)$ (Table I). Response rates and participant age stratified into 10-year age groups are reported in Table II. The 25-34 group was the age group with the lowest response rate in all 6 years examined, whereas higher rates were observed among women aged $35-44$ and $45-54$ years: $31.4 \%$ in 2008 , 
Table I. Organized screening data between two 3-year periods.

\begin{tabular}{|c|c|c|c|c|c|c|c|}
\hline Year & Target population, $\mathrm{n}$ & Total invited, $\mathrm{n}$ & Respondents, $\mathrm{n}$ & Total, \% & Non-respondents, $\mathrm{n}$ & Total, \% & P-value \\
\hline $2008-2010$ & $85,289^{a}$ & 36,581 & 14,142 & 38.7 & 22,439 & 61.3 & $<0.001$ \\
\hline 2008 & & 16,222 & 4,500 & 27.7 & 11,722 & 72.3 & \\
\hline 2009 & & 12,042 & 5,368 & 44.6 & 6,674 & 55.4 & \\
\hline 2010 & & 8,317 & 4,274 & 51.4 & 4,043 & 48.6 & \\
\hline 2011-2013 & $85,764^{b}$ & 60,118 & 24,206 & 40.3 & 35,912 & 59.7 & $<0.001$ \\
\hline 2011 & & 21,434 & 7,529 & 35.1 & 13,905 & 64.9 & \\
\hline 2012 & & 20,576 & 9,015 & 43.8 & 11,561 & 56.2 & \\
\hline 2013 & & 18,108 & 7,662 & 42.3 & 10,446 & 57.7 & \\
\hline
\end{tabular}

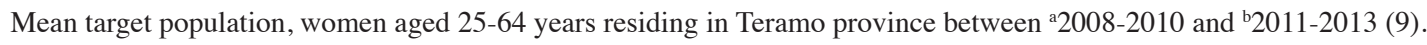

Table II. Distribution of the respondents by age class and year of participation.

\begin{tabular}{lrrrrr}
\hline \multicolumn{5}{c}{ Age groups of respondents (years) } \\
Yyyr & $25-34, \mathrm{n}(\%)$ & $35-44, \mathrm{n}(\%)$ & $45-54, \mathrm{n}(\%)$ & $55-64, \mathrm{n}(\%)$ & Total, n \\
\hline 2008 & $913(20.3)$ & $1,413(31.4)$ & $1,225(27.2)$ & $949(21.1)$ & 4,500 \\
2009 & $905(16.9)$ & $1,479(27.6)$ & $1,556(29.0)$ & $1,428(26.6)$ & 5,368 \\
2010 & $538(12.6)$ & $1,069(25.0)$ & $1,375(32.2)$ & $1,292(30.2)$ & 4,274 \\
2011 & $1,368(18.2)$ & $2,309(30.7)$ & $2,181(29.0)$ & $1,671(22.2)$ & 7,529 \\
2012 & $1,655(18.4)$ & $2,438(27.0)$ & $2,776(30.8)$ & $2,146(23.8)$ & 9,015 \\
2013 & $1,495(19.5)$ & $2,296(30.0)$ & $2,260(29.5)$ & $1,611(21.0)$ & 7,662 \\
Total & $6,874(100.0)$ & $11,004(100.0)$ & $11,373(100.0)$ & $9,097(100.0)$ & 38,348 \\
\hline
\end{tabular}

Table III. Human papillomavirus (HPV) tests repeated at 1-year in women with positive-HPV and negative test triage cytology, according to age groups and years.

\begin{tabular}{|c|c|c|c|c|c|}
\hline \multirow[b]{2}{*}{ Year } & \multicolumn{4}{|c|}{ Age groups (years) } & \multirow[b]{2}{*}{ All cases, $n$} \\
\hline & $25-34, \mathrm{n}(\%)$ & $35-44, \mathrm{n}(\%)$ & $45-54, \mathrm{n}(\%)$ & $55-64, \mathrm{n}(\%)$ & \\
\hline 2011 & $105(28.7)$ & $112(30.6)$ & $90(24.6)$ & $59(16.1)$ & 366 \\
\hline 2012 & $124(35.5)$ & 94 (26.9) & $82(23.5)$ & $49(14.0)$ & 349 \\
\hline 2013 & $92(35.9)$ & $76(29.7)$ & $57(22.2)$ & $31(12.1)$ & 256 \\
\hline All cases & $321(33.0)$ & $282(29.0)$ & $229(23.6)$ & $139(14.3)$ & 971 \\
\hline
\end{tabular}

$30.7 \%$ in 2011 and $30.0 \%$ in 2013 for the former group and $29.0 \%$ in $2009,32.2 \%$ in 2010 and $30.8 \%$ in 2012 for the latter $(\mathrm{P}<0.001)$.

Finally, the HPV tests repeated at 1-year in women with positive-HPV/negative test triage cytology were evaluated (Table III).

Diagnostic categories. The diagnostic categories detected by cytology and the histological results are reported in Table IV. Organized screening used the Pap test as the primary screening test between 2008 and 2010 and the HPV DNA test followed by cytology triage in HPV-positive samples between 2011 and 2013. Spontaneous screening always involved the Pap test. Negative tests over the 6 years of the study were 35,029 (91.3\%) among women undergoing organized screening and 18,595 (87.9\%) among those undergoing spontaneous screening. Positive tests throughout the study were 4,542 (7.6\%) and 1,997 (5.2\%) (the tests with HPV-positive and negative Pap test were considered separately) for organized screening, and 2,545 (12.0\%) for spontaneous screening. With regard to the diagnostic category, there was a significant difference in HSIL, LSIL and undetermined lesion diagnoses provided by organized and spontaneous screening (Table IV). There were 50.5 vs. $49.5 \%$ HSIL, 42.8 vs. $57.2 \%$ LSIL, 57.5 vs. $42.5 \%$ AGC and 54.2 vs. $45.8 \%$ ASC-US, respectively (all $\mathrm{P}<0.001$ ). Adoption of the HPV test between 2011 and 2013 induced a significant reduction in the number of cytological lesions found in the organized programme 

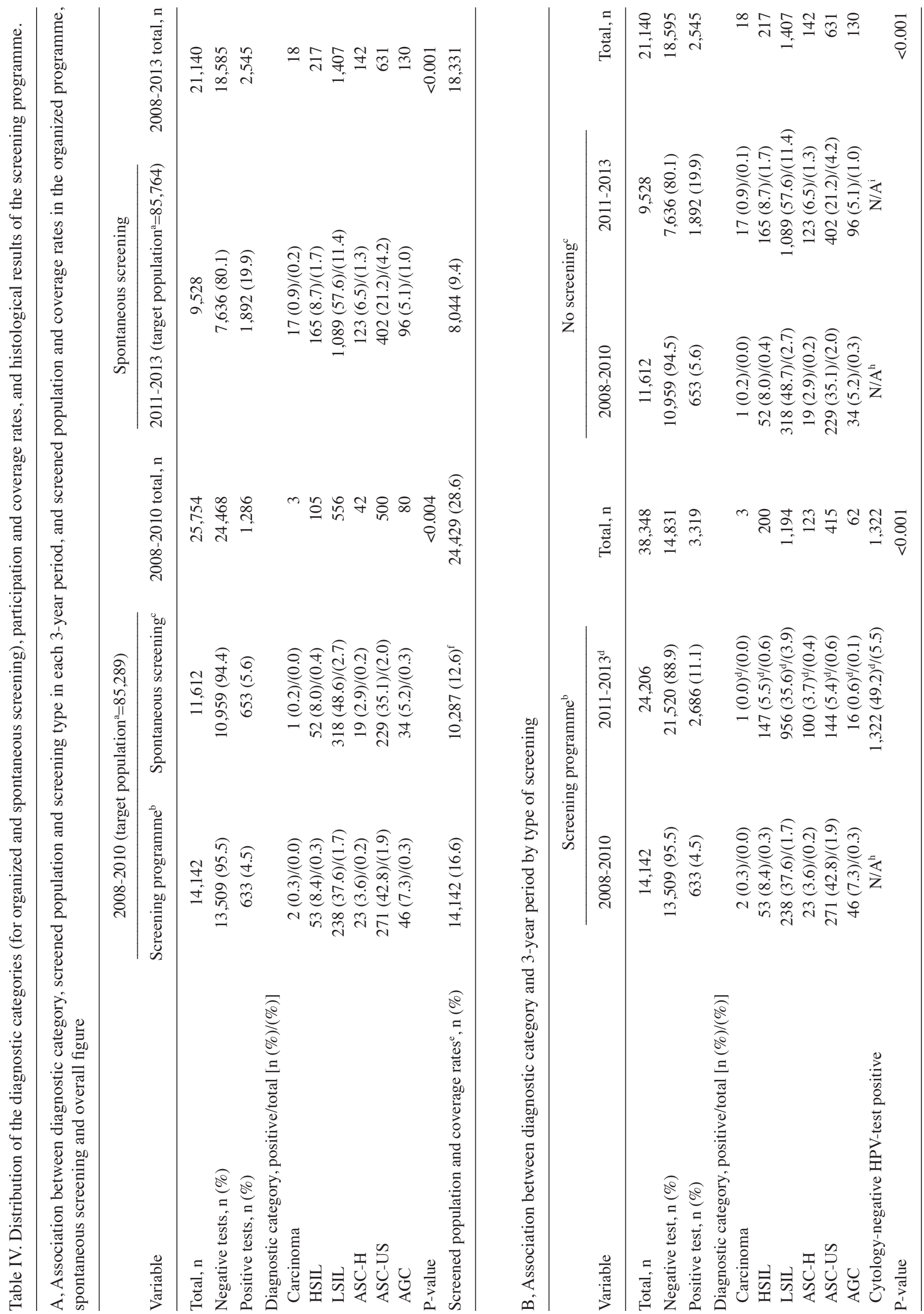


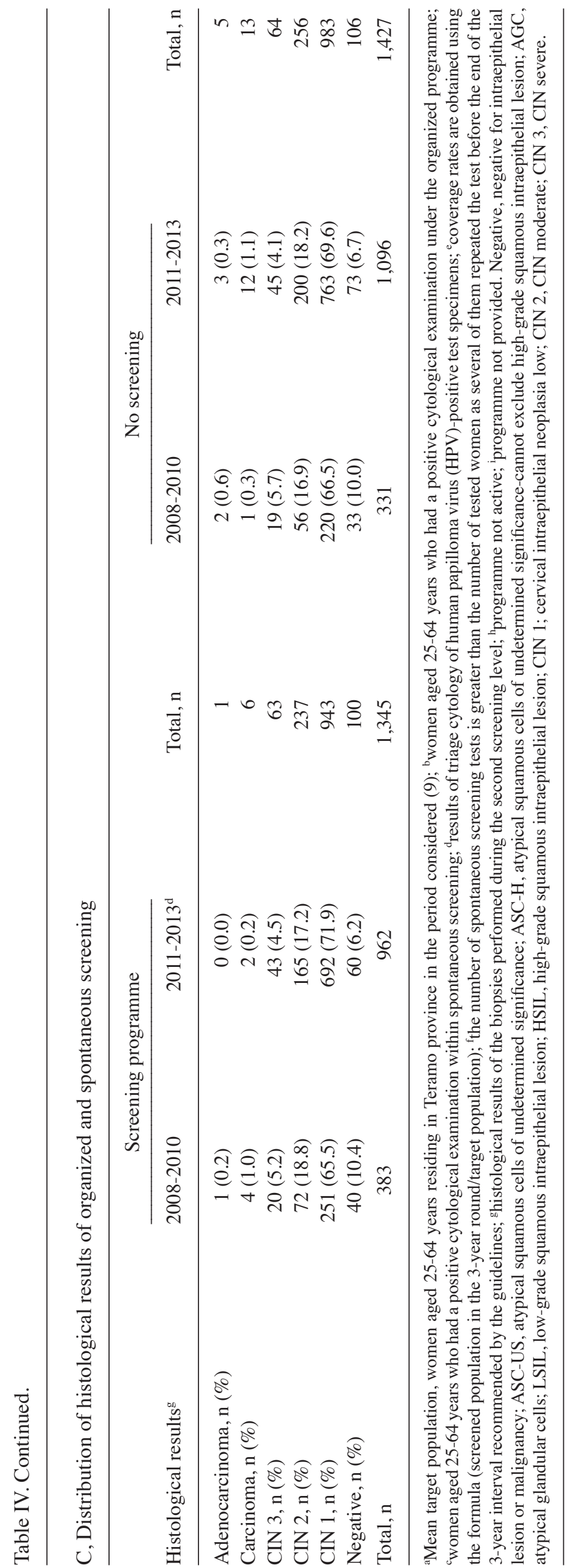

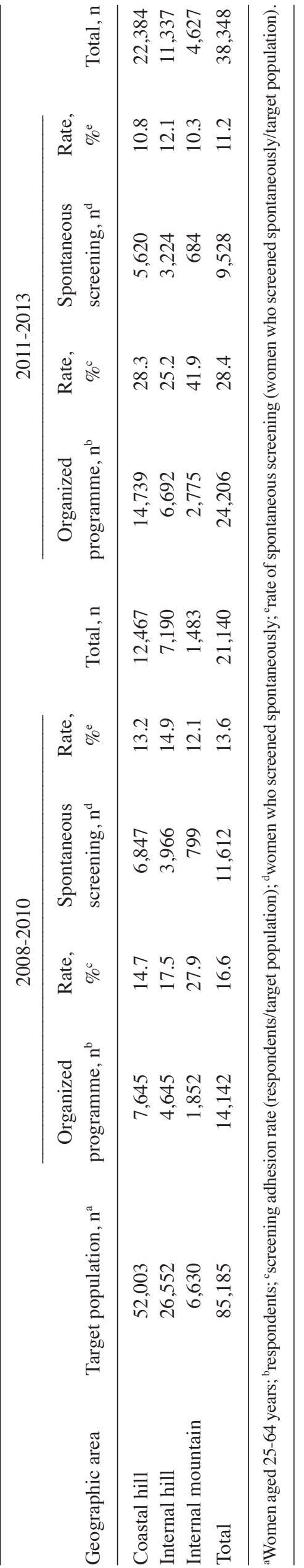


compared with the spontaneous testing; 47.1 vs. $52.9 \%$ HSIL, 44.8 vs. $55.2 \%$ ASC-H, 46.7 vs. $53.2 \%$ LSIL, 14.3 vs. $85.7 \%$ AGC and 26.4 vs. $73.6 \%$ ASC-US, respectively, also as the majority of the spontaneous tests were performed in private gynaecological practices where symptomatic women are usually examined (all $\mathrm{P}<0.001$ ). Notably, the tests from the programme were more than double those from spontaneous testing. In Table IV, the diagnostic categories detected in the two 3-year periods are divided into those detected by organized and spontaneous screening. Their subdivision highlights the effect of the introduction of the HPV test in the organized programme. The number of positive tests increased from $4.5 \%$ (633/14.142) between 2008 and 2010 to $5.6 \%$ (1,364/24,206). However, the cytological lesions detected in the two periods had a different distribution. In particular, in the second period there were significantly fewer diagnoses of undetermined lesions (ASC-US, from 65.3 to $34.7 \%$; AGC, from 74.2 to $25.8 \%$ ) and significantly more HSIL (from 25.5 to $73.5 \%$ ) and LSIL (from 19.9 to $81.3 \%$ ) (all $\mathrm{P}<0.001$ ). The number of cytological lesions detected in women screening spontaneously also increased from 5.6\% $(653 / 11,612)$ between 2008 and 2010 to $19.9 \%(1,892 / 9,528)$ between 2011 and 2013. However the increase was distributed over all the diagnostic categories $(\mathrm{P}<0.001)$. The second screening level involved 2,772 biopsies, $60.5 \%$ of positive cytological tests, which are reported in Table IV and are stratified by histological result and divided into screening programme and spontaneous screening in the two 3-year periods. A larger number of preneoplastic histological lesions (CIN) were detected in the second 3-year period compared with the first. The increase in CIN 1 lesions is the consequence of the adoption of the HPV test, which detects HPV infection, whereas the increased number of CIN 2 and CIN 3 lesions may constitute a diagnostic improvement due to the new technique.

Geographic distribution. The geographic distribution of women undergoing organized and spontaneous screening in the two 3-year periods is reported in Table V. Between 2008 and 2011, the participation rates were similar for the two types of screening in coastal hill (14.7 vs. 13.2\%) and internal hill areas (17.5 vs. $14.9 \%)$, where the major municipalities are located, whereas in internal mountain areas, respondents to the programme were more than twice the number of women screening spontaneously (27.9 vs. $12.1 \%)(\mathrm{P}<0.001)$. Between 2011 and 2013, the rates of spontaneous screening decreased significantly in all areas, but the largest reduction was in the internal mountain areas (41.9 vs. $10.3 \%$ ), followed by coastal hill (28.3 vs. $10.8 \%)$ and internal hill areas (25.2 vs. $12.1 \%)$ (all $\mathrm{P}<0.001$ ).

\section{Discussion}

The CC screening programme of Teramo province has undergone significant changes from the first to the second of the two 3-year periods examined herein, in terms of the test used and the organization. The Pap test, employed between 2008 and 2010, was replaced between 2011 and 2013 with the HPV test, together with triage cytology in positive or suspicious cases. Furthermore, to reduce spontaneous screening and increase participation in the organized programme the
Abruzzo's regional government subjected free CC screening to the organized programme through the invitation letter in 2011. The marked reduction in screening activity identified in 2010 is possibly due to the change to the HPV test and the changes to the SIW required to activate the new screening protocol, which likely reduced the invitations letters sent in the second half of 2010. The requirement of the invitation letter to screen for free determined a strong reduction in spontaneous screening at public facilities between 2011 and 2013.

Nonetheless the response rate to the second 3-year programme was not commensurate to the number of invitations sent, indicating the requirement for corrective measures to increase adhesion. Subdivision of participants into 10-year age groups showed that young women (25-34 years) had lower response rates to the invitation to screen, in line with national and international data $(12,13)$. A notable finding was the significantly different frequency distribution of cytological lesions into diagnostic categories between organized and spontaneous screening, particularly when the two 3-year periods were compared. The present data show a greater frequency of negative tests among women undergoing organized screening, due to the fact that the programme invites women irrespective of their state of health, whereas spontaneous screening (at gynaecological practices or gynaecology departments) is more likely to involve a larger number of symptomatic women, who may have cytological lesions. More cancer lesions were detected in the spontaneous population $(n=18)$ compared to the population under screening $(\mathrm{n}=3)$.

Between 2008 and 2010, a similar number of lesions belonging to the various diagnostic categories were detected by organized and spontaneous screening and involved a similar frequency of referral to second-level examinations $(49.2$ vs. $50.8 \%)$. In particular, after stratification by year a similar distribution of cases was observed as follows: 213 (33.6) vs. $218(33.4 \%)$ in 2008; 209 (33.0) vs. 215 (32.9\%) in 2009; and 211 (33.3) vs. 210 (32.2\%) in 2010.

The introduction of the HPV test and cytology triage for positive samples between 2011 and 2013 (14) enhanced the diagnostic ability of the organized programme. A comparison of cytological lesion frequency in the various diagnostic categories demonstrated a significant difference $(\mathrm{P}<0.001)$ between the two 3-year periods of the organized programme, with a strong reduction in the diagnosis of undetermined lesions and earlier identification of potentially severe cytological lesions in the second round. By contrast, the frequency of lesions, particularly that of undetermined lesions, increased significantly $(\mathrm{P}<0.001)$ from the first to the second 3-year period in women who were screened spontaneously, resulting in a disproportionate number of second-level colposcopies that was not warranted by actual requirement. The increased number of high-grade histological lesions identified between 2011 and 2013 in the organized programme is to be viewed as early diagnosis of existing cytological lesions due to the HPV test. In previous randomized trials using the HPV test and cytology triage $(15,16)$, such an increment was followed by a reduction in the high-grade cytological and histological lesions diagnosed in subsequent rounds and extended the low-risk period. These data now enable the interval between tests to be extended from 3 to 5 years, thus improving the cost-effectiveness of organized programmes (17). A positive-HPV test when there are small, undetermined 
cellular lesions makes an LSIL diagnosis possible, thus significantly reducing ASC-US diagnoses. Analysis of screening rates by geographic area and by period demonstrated lower rates of spontaneous screening in internal mountain areas, possibly due to the small number of gynaecological practices, and markedly higher rates in the other areas, where municipalities are larger and private gynaecological practices are more numerous. However, it should be noted that following the organizational changes introduced by the regional government of Abruzzo, whereby free testing is subjected to the invitation to screen, spontaneous screening was reduced between 2011 and 2013 in all geographic areas, reflecting the effectiveness of the provision in recruiting women to the organized programme. The present data demonstrate an increase in coverage from the first to the second 3-year period and a reduction in spontaneous screening participation. However, increasing screening coverage remains a priority in this region (18).

In conclusion, the decision to subject free testing to the screening invitation has achieved rapid effects. Participants in the organized programme almost doubled between 2011 and 2013 compared with between 2008 and 2010. Even though the first round with the HPV test has produced an increase in the number of high-grade cytological lesions detected, the test will enable the interval between rounds to be extended, thus enhancing programme cost-effectiveness. Furthermore, the reduction in the diagnoses of undetermined lesions will reduce referrals for second-level testing, thus reducing laboratory workloads and the emotional burden on women.

\section{Acknowledgements}

The authors would like to thank Dr Tatiana Reggi (Pathological Anatomy, AUSL 4 Teramo) for her collaboration in the current study and for the support in the initial database construction.

\section{References}

1. Bray F, Ren JS, Masuyer E and Ferlay J: Global estimates of cancer prevalence for 27 sites in the adult population in 2008. Int J Cancer 132: 1133-1145, 2013.

2. GLOBOCAN 2012 (IARC) Section of Cancer Information. http:// globocan.iarc.fr/Pages/fact_sheets_population.aspx. Accessed September 15, 2014.

3. AIRTUM-Italian SocietyCancer Registries. I numeridel cancro in Italia 2013. http://www.registri-tumori.it/cms. Accessed September 19, 2014.

4. Capocaccia R, Martina L, Inghelmann R, Crocetti E, De Lisi V, Falcini F, Guzzinati S, Rosso S, Tagliabue G, Tumino R, et al: A method to estimate mortality trends when death certificates are imprecisely coded: An application to cervical cancer in Italy. Int J Cancer 124: 1200-1205, 2009.
5. Giorgi Rossi P, Camilloni L, Cogo C, Federici A, Ferroni E, Furnari G, Giordano L, Grazzini G, Iossa A, Jimenez B, et al: Methods to increase participation in cancer screening programmes. Epidemiol Prev 36 (1 Suppl 1): S1-S104, 2012.

6. CCM Italy-Centro Controllo malattie, ministero della Salute. Dieci annidi programmidi screening in Italia. Roma Zadig ed 2012. http://www.osservatorionazionalescreening.it/sites/default/ files/allegati/Brochure_10anni.pdf. Accessed September 20, 2014.

7. ONS 2014. I programmidi screening in Italia. Rapporto ONS 2014. Roma. Zadig ed. 2014. http://www.osservatorionazionalescreening.it/sites/default/files/allegati/Screening_2014_web.pdf (accessed 02 September 2014).

8. Altobelli E and Lattanzi A: Cervical carcinoma in the European union: An update ondisease burden, screening program state of activation, and coverage as of march 2014. Int J Gynecol Cancer 25: 474-483, 2015.

9. ISTAT. Istituto Nazionale di Statistica-Italia. http://demo.istat.it/. Accessed October 10, 2014.

10. Arbyn M, Anttila A, Jordan J, Ronco G, Schenck U, Segnan N, Wiener H, Herbert A and von Karsa L: European guidelines for quality assurance in cervical cancer screening. Second edition-summary document. Ann Oncol 21: 448-458, 2010.

11. Wright TC Jr, Cox JT, Massad LS, Twiggs LB and Wilkinson EJ; ASCCP-Sponsored Consensus Conference: 2001 Consensus guidelines for the management of women with cervical cytological abnormalities. JAMA 287: 2120-2129, 2002.

12. Berardi R, Nacciarriti D, Tamburrano T, Carbonari G, Romagnoli E, Duca M, Burattini M, Silva RR, Cellerino R and Cascinu S; Women's Council of the City of Senigallia, Ancona, Italy: Compliance with breast and cervical cancer screening programs in women: Results from a population-based study. Tumori 99: 565-571, 2013

13. Waller J, Jackowska M, Marlow L and Wardle J: Exploring age differences in reasons for nonattendance for cervical screening: A qualitative study. BJOG 119: 26-32, 2012.

14. Cox JT, Castle PE, Behrens CM, Sharma A, Wright TC Jr and Cuzick J; Athena HPV Study Group: Comparison of cervical cancer screening strategies incorporating different combinations of cytology, HPV testing, and genotyping for HPV 16/18: Results from the ATHENA HPV study. Am J Obstet Gynecol 208: 184. e1-e184.e11, 2013.

15. Ronco G, Giorgi-Rossi P, Carozzi F, Confortini M, Dalla Palma P, Del Mistro A, et al: Efficacy of human papillomavirus testing for the detection of invasive cervical cancers and cervical intraepithelial neoplasia: A randomised controlled trial. Lancet Oncol 11: 249-257, 2010.

16. Rijkaart DC, Berkhof J, Rozendaal L, van Kemenade FJ, Bulkmans NW, Heideman DA, Kenter GG, Cuzick J, Snijders PJ and Meijer CJ: Human papillomavirus testing for the detection of high-grade cervical intraepithelial neoplasia and cancer: Final results of the POBASCAM randomised controlled trial. Lancet Oncol 13: 78-88, 2012

17. Goldhaber-Fiebert JD, Stout NK, Salomon JA, Kuntz KM and Goldie SJ: Cost-effectiveness of cervical cancer screening with human papillomavirus DNA testing and HPV-16,18 vaccination. J Natl Cancer Inst 100: 308-320, 2008.

18. Giorgi Rossi P, Fortunato C, Barbarino P, Boveri S, Caroli S, Del Mistro A, Ferro A, Giammaria C, Manfredi M, Moretto T, et al: Self-sampling to increase participation in cervical cancer screening: An RCT comparing home mailing, distribution in pharmacies, and recall letter. Br J Cancer 112: 667-675, 2015 